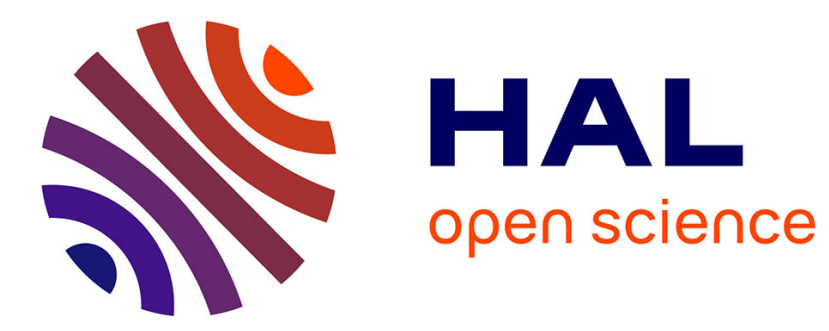

\title{
Singularity analysis of digital signals through the evaluation of their Unpredictable Point Manifold
}

Oriol Pont, Antonio Turiel, Hussein Yahia

\section{To cite this version:}

Oriol Pont, Antonio Turiel, Hussein Yahia. Singularity analysis of digital signals through the evaluation of their Unpredictable Point Manifold. International Journal of Computer Mathematics, 2013, 90 (8), pp.1693-1707. 10.1080/00207160.2012.748895 . hal-00688715

HAL Id: hal-00688715 https://hal.inria.fr/hal-00688715

Submitted on 10 Dec 2012

HAL is a multi-disciplinary open access archive for the deposit and dissemination of scientific research documents, whether they are published or not. The documents may come from teaching and research institutions in France or abroad, or from public or private research centers.
L'archive ouverte pluridisciplinaire $\mathbf{H A L}$, est destinée au dépôt et à la diffusion de documents scientifiques de niveau recherche, publiés ou non, émanant des établissements d'enseignement et de recherche français ou étrangers, des laboratoires publics ou privés. 
International Journal of Computer Mathematics

Vol. 00, No. 00, Month 200x, 1-12

\title{
RESEARCH ARTICLE
}

\section{Singularity analysis of digital signals through the evaluation of their Unpredictable Point Manifold}

\author{
Oriol Pont ${ }^{\mathrm{a} *}$, Antonio Turiel ${ }^{\mathrm{b}}$ and Hussein Yahia ${ }^{\mathrm{a}}$ \\ a INRIA Bordeaux Sud-Ouest \\ 351 Cours de la Libération bt. A29bis, 33405 Talence, France \\ b Institut de Ciències del Mar, ICM - CSIC \\ Passeig marítim de la Barceloneta 37-49, 08003 Barcelona, Catalonia, Spain
}

( )

\begin{abstract}
The local singularity exponents of a signal are directly related to the distribution of information in it. This fact implies that accurate evaluation of such exponents opens the door to signal reconstruction and characterisation of the dynamical parameters of the process originating the signal. Many practical implications arise in a context of digital signal processing, since the information on singularity exponents is usable for compact encoding, reconstruction and inference. Since singularity exponents are conceptually associated to differential calculus, its evaluation in a digital context is not straightforward and it requires the calculation of the Unpredictable Point Manifold of the signal. In this paper, we present an algorithm for estimating the values of singularity exponents at every point of a digital signal of any dimension. We show that the key ingredient for robust and accurate reconstructibility performance lies on the definition of multiscale measures in the sense that they encode the degree of singularity and the local predictability at the same time.
\end{abstract}

Keywords: multiscale measures, reconstructible systems, MSC-94A08, MSC-43A32, MSC-46T12, CCS-G.1.m

\section{Introduction}

In digital signals of all kinds, the estimation of their local singularity exponents provides a valuable information that can be used to compactly encode the signal values $[16,17]$. Furthermore, the singularity exponents can be accurately estimated at the top points of a Wavelet Transform Modulus Maxima (WTMM) line, for the case of turbulent flows or analogous scale-invariant systems $[15,18,19]$. However, the generalization of this methodology from the WTMM points to any point of the signal (at its finest resolution level) is not straightforward: such generalizations become convoluted and they provide rather imprecise estimates of singularity exponents outside the WTMM lines [29] and poor description of their distributions [36].

There is a completely different approach based on local gradient modulus measures that are numerically estimated by convolution with wavelet or other positive multiscale kernels. This method provides more stable and accurate results for discretised signals even at maximum resolution $[30,34,38]$. This approach provides the basic bricks of the so-called Microcanonical Multiscale Formalism (MMF) [22, 37], which gives access to unveil key physical descriptors for turbulence and turbulent-like systems [10, 21, 23, 24, 35, 37, 39, 40]. This way, estimating the singularity exponents of a signal also provides information about the physical process behind it.

At the local level, the accuracy of MMF-based singularity-analysis estimates is significantly sensitive to the shape of the multiscale measure used to obtain them [37], even if the estimated distributions are not [36]. Therefore, if we want to characterize only the statistical properties of the singularity exponents we can project the signal over any well-behaved kernel, such as positive multiscale functions with fast enough decay [30], but if we need a precise geometrical identification of the singularity structures and their underlying patterns from edges to textures, then we require a fine-tuned multiscale measure.

\footnotetext{
${ }^{*}$ Corresponding author: oriol.pont@inria.fr
} 
The work we present in the following describes a method that allows high-performance determination of the singularity exponents, with an algorithm that is fast, computationally cheap, stable, accurate and provides fine resolution. Its definition relies in the connection of singularity exponents with the concept of signal reconstruction from the Unpredictable Point Manifold (UPM). Singularity analysis establishes a link between singularity exponent and reconstructibility degree of a point, in a sense that the UPM can be estimated as the Most Singular Component (MSC) of the signal [32].

To validate the detection of the information content provided by the singularity exponents as calculated from UPM-measures, we illustrate a comparison of them with the unrelated method of multiscale entropy [5, 28]. We observe that all the features and their informative degree coincide and furthermore the singularity exponents provide a finer resolution and so a better localization of the information.

This paper is presented as an extended version of our previous findings that we have presented at the 14th International Workshop on Combinatorial Image Analysis, IWCIA 2011 held in Madrid in May 2011 [25]. Here we summarise our previous findings, while at the same time we expand them accordingly: we give a deeper description of the analysis performed and the methods used, and we present a more exhaustive evaluation (which includes both signal reconstructibility and illustrating a comparison of information content with multiscale entropy). Namely, since analysis of multidimensional signals may also be of interest to the reader, we present here a generalisation of our technique to $\mathrm{nD}$ and give a wider insight to it by illustrating the singularity analysis and reconstruction performance for the cases of empirical signals of 1D (stock-market series), 2D (Meteosat IR map) and 3D (ERA-40 global climate re-analysis).

The paper is structured as follows: in the next Section we introduce the basics of singularity analysis. Section 3 explains the concept of reconstruction from the MSC. Section 4 is the core of the paper: first the conditions defining UPM-measures are discussed, then Subsection 4.1 explains the definitions for calculus on reduced neighbourhoods through the cross-gradient and cross-reconstruction operators, and finally in Subsection 4.2 our algorithm to retrieve the UPM and thus the singularity exponents from digital signals is presented. Some analyses on empirical signals of 1D, 2D and 3D are shown in Section 5. The last section, Section 6, presents the conclusions of our work.

\section{Evaluating singularity exponents}

In mathematical analysis, the concept of singularity exponents refers to the analysis of singularities of differentiable functions in a broad sense and different non-equivalent defintions are possible; in the present work we focus on its usual meaning in the theory of complex systems. Using singularity analysis we intend to characterise the local behaviour of a $\mathbb{R}^{m}$-valued function $f(\mathbf{x})$ defined on $\mathbb{R}^{d}$ around each one of its domain points $\mathbf{x}$ according to their so-called singularity exponents, Hölder exponents [11] or Hurst exponents $[12,27]$. If the signal behaves at point $\mathbf{x}$ according to:

$$
\|f(\mathbf{x}+\mathbf{r})-f(\mathbf{x})\|=\alpha(\mathbf{x}) r^{h(\mathbf{x})}+o\left(r^{h(\mathbf{x})}\right) \quad(\mathbf{r} \rightarrow 0)
$$

then $h(\mathbf{x})$ is the singularity exponent at $\mathbf{x}$ : small perturbations around $\mathbf{x}$ lead to changes in the function value as a power of the displacement modulus $r=\|\mathbf{r}\|$. A strictly $n$-times derivable function naturally leads to an integer Hölder exponent $h(\mathbf{x})=n$. As a result, this formulation allows to generalise the concept of integer differentiability to fractional differentiability, but to complete that transposition, a slightly more exigent formulation of eq. (1) is required: we need to assume that there exists a $(1,1)$ continuous tensor from $\mathbb{R}^{d}$ to $\mathbb{R}^{m}, \alpha(\mathbf{x})$, such that

$$
f(\mathbf{x}+\mathbf{r})-f(\mathbf{x})=\langle\alpha(\mathbf{x}) \mid \mathbf{r}\rangle r^{h(x)-1}+o\left(r^{h(\mathbf{x})}\right) \quad(\mathbf{r} \rightarrow 0)
$$

where $\langle\alpha(\mathbf{x}) \mid \mathbf{r}\rangle$ is the standard duality bracket for $(1,1)$ tensors. If such representation is possible, the exponent $h(\mathbf{x})$ is called the Hurst exponent of the function, but if only eq. (1) applies then we use the term Hölder exponent.

In real-world empirical signals, Hölder exponents often appear masked by long-range correlations and fluctuations due to noise and discretisation effects that obstruct their direct estimation [34, 37]. A generalization that avoids such problems consists of defining singularity exponents from gradient measures [34]. Let $s(\mathbf{x})$ be a signal defined in $\mathbb{R}^{d}$ with values in $\mathbb{R}$, we define the gradient-based measure $\mu$ through its density $\mathrm{d} \mu(\mathbf{x})$ :

$$
\mathrm{d} \mu(\mathbf{x})=\|\nabla s\|(\mathbf{x}) \mathrm{d} \mathbf{x}
$$

which is defined as absolutely continuous with respect to the Lebesgue measure. As a consequence, the measure of any Borelian $\mathcal{A}$ is [37]: 


$$
\mu(\mathcal{A})=\int_{\mathcal{A}} \mathrm{d} \mathbf{x}\|\nabla s\|(\mathbf{x})
$$

The main advantage of gradient measures is that they characterise the local degree of singularity of the signal [34]. From eq. (1), let us now consider a function $f(\mathbf{x})$ that has $h(\mathbf{x})+1$ as Hölder exponents (whith a +1 shift introduced for later convenience). Let $\mathcal{B}_{r}(\mathbf{x})$ be a ball of radius $r$ (for some arbitrary norm in $\mathbb{R}^{d}$ ) centred around $\mathbf{x}$. This measure behaves as:

$$
\mu\left(\mathcal{B}_{r}(\mathbf{x})\right)=\alpha(\mathbf{x}) r^{d+h(\mathbf{x})}+o\left(r^{d+h(\mathbf{x})}\right)(r \rightarrow 0)
$$

where $d$ is the dimension of the signal domain. For discrete signals, we can interpolate this measure by means of wavelet projections $[13,14]$. Let $\Psi$ be a wavelet function, $\mathcal{T}_{\Psi} \mu(\mathbf{x}, r)$ is the projection of the measure $\mu$ at the point $\mathbf{x}$ and scale $r[6,15]$ :

$$
\mathcal{T}_{\Psi} \mu(\mathbf{x}, r)=\int_{\mathbb{R}^{d}} \mathrm{~d} \mu\left(\mathbf{x}^{\prime}\right) \frac{1}{r^{d}} \Psi\left(\frac{\mathbf{x}-\mathbf{x}^{\prime}}{r}\right)
$$

This way, $\mathcal{T}_{\Psi}$ is an operator that maps from a set $\mathcal{M}$ of $\sigma$-finite measures on $\mathbb{R}^{d}$ to the set of $\mathbb{R}^{d} \times \mathbb{R}^{+} \rightarrow \mathbb{R}$ functions. The singularity exponents of the signal as per eq. (5) are directly expressed on the wavelet projection too $[6,34]$ :

$$
\mathcal{T}_{\Psi} \mu(\mathbf{x}, r)=\alpha_{\Psi}(\mathbf{x}) r^{h(\mathbf{x})}+o\left(r^{h(\mathbf{x})}\right)(r \rightarrow 0)
$$

with the same singularity-exponent values $h(\mathbf{x})$.

Measures can then be used on discretised data with any multiscale function $\Psi$ in eq. (7), without any requirement of the admissibility property [37]. As the attainable resolution level depends on the number of zero-crossings of the wavelet used [36], hence we can even use positive kernels that maximise the resolution at which we estimate the singularity exponents $[30]$.

\section{A reconstruction formula from the most singular set}

Signal reconstructibility from a partial set of its gradient is the key concept to define a wavelet that precisely identifies the singularity exponents. The theoretical development and its practical implementation give raise to a reconstruction algorithm [32] relating reconstructibility to singularity.

We require that every point of the signal has a singularity exponent defined $[8,20]$ and that these exponents organise in sets that follow the signal multiscale structures with a geometrical hierarchy [7]. As a consequence, such structures follow cascading relations whose distribution matches those of the macroscopic descriptors of the system under study [26]. Since it is difficult for classical methods to consistently assess accurate values of singularity exponents on empirical signals, these structures have been often characterised in a statistical way only. However, it is possible to have a direct access to this geometrical hierarchy through the reconstruction of the full structure from its vertex [32]. In multifractal signals, the set associated to the vertex is formally well-known and it corresponds to the Most Singular Component (MSC), i.e., the set of points having the most singular values of singularity exponent $[34,37]$.

For the gradient of a signal over the MSC to fully reconstruct the signal, it needs to concentrate all its information [32]. We look for a universal operator able to reconstruct a signal that follows the known statistical symmetries of turbulent-like and multiscale signals [8]. Such operator, if it exists, it is uniquely defined under the requirements of being deterministic, linear, translational invariant, isotropic and leading to the known power-spectrum shape.

Given a multiscale signal $s, \mathcal{F}_{\infty}$ is its MSC: $\mathcal{F}_{\infty}$ is the set of points $\mathbf{x}$ such that $h(\mathbf{x}) \in] h_{\infty}-\Delta, h_{\infty}+\Delta\left[\right.$ with $h_{\infty}$ being the minimum value of $h(\mathbf{x})$ over the finite domain of the discrete signal and $\Delta$ is an ideally infinitessimal threshold parameter. Let $\nabla_{\mathcal{F}} s$ be the essential gradient of $s$ defined as:

$$
\nabla_{\mathcal{F}} s(\mathbf{x})=\nabla s(\mathbf{x}) \delta_{\mathcal{F}}(\mathbf{x})
$$


where $\delta_{\mathcal{F}_{\infty}}$ consists of a delta distribution on the continuum of the $\mathcal{F}_{\infty}$ : a distribution that is homogeneous in Hausdorff topological dimension to a repartition in between dimensions $d-1$ and $d$, so that it assigns a uniform weight inside the MSC points $\mathcal{F}_{\infty}$ while it vanishes outside.

If we denote the gradient reconstructing functional by $\mathcal{G}$, a deterministic representation of the gradient of the signal means:

$$
\nabla s(\mathbf{x})=\mathcal{G}\left(\nabla_{\mathcal{F}_{\infty}} s(\mathbf{x})\right)
$$

Considering now the fact of $\mathcal{G}$ being linear, an integral representation is given by:

$$
\nabla s(\mathbf{x})=\int_{\mathcal{F}_{\infty}} \mathrm{d} \mathbf{x}^{\prime} \mathbb{G}\left(\mathbf{x}, \mathbf{x}^{\prime}\right) \nabla s\left(\mathbf{x}^{\prime}\right)
$$

The property of translational invariance implies:

$$
\nabla s(\mathbf{x})=\int_{\mathcal{F}_{\infty}} \mathrm{d} \mathbf{x}^{\prime} \mathbb{G}\left(\mathbf{x}-\mathbf{x}^{\prime}\right) \nabla s\left(\mathbf{x}^{\prime}\right)
$$

where $\mathbb{G}$ is the $\mathbb{R}^{d} \times \mathbb{R}^{d}$ density of the linear operator $\mathcal{G}$. By considering the differential forms involved, since $\nabla s(\mathbf{x})$ is a gradient in the distributional sense, $\mathbb{G}$ can also be represented as a gradient of some functional-vector $\vec{g}$ :

$$
s(\mathbf{x})=\int_{\mathcal{F}_{\infty}} \mathrm{d} \mathbf{x}^{\prime} \vec{g}\left(\mathbf{x}-\mathbf{x}^{\prime}\right) \cdot \nabla s\left(\mathbf{x}^{\prime}\right)
$$

which actually is a convolution between $\vec{g}$ and $\nabla_{\mathcal{F}} s$, eq. (8), expressed in the Fourier domain as:

$$
\hat{s}(\mathbf{k})=\hat{\vec{g}}(\mathbf{k}) \cdot \widehat{\nabla_{\mathcal{F}}} s(\mathbf{k})
$$

which is the reconstruction formula [32].

The vector field $\vec{g}$ is the universal reconstruction kernel. As mentioned, we require that it is isotropic and does not alter the power spectrum (e.g., a $f^{-2}$ signal has a white gradient). Isotropy means it has the same direction as the unit vector of the frequency, namely: $\hat{\vec{g}}(\mathbf{k})=\hat{g}(k) \mathbf{u}_{\mathbf{k}}$ where $k$ is the frequency modulus: $\mathbf{k}=k \mathbf{u}_{\mathbf{k}}$. Then the modulus of the reconstruction kernel, $\hat{g}(k)$, is:

$$
\hat{\vec{g}}(\mathbf{k})=i \frac{\mathbf{u}_{\mathbf{k}}}{k}
$$

with $i=\sqrt{-1}$ as the imaginary unit, as we require it to work also in the case where the MSC is the whole domain. This definition implies that the reconstruction kernel $\vec{g}$ is actually an inverse gradient operator. The reconstruction is always perfect if we consider the whole domain, i.e., if $\mathcal{F}_{\infty}=\mathbb{R}^{d}$ then eq. (13) reduces to a trivial identity: $\nabla_{\mathcal{F}} s=\nabla s$. It is not evident at all if there exists a smaller set $\mathcal{F}_{\infty} \subset \mathbb{R}^{d}$ for which eq. (13) returns a perfect reconstruction, at least without assuming any further conditions. Nevertheless, any set $\mathcal{F}$ that leads to a perfect reconstruction with the reconstruction formula verifies that [32]:

$$
\operatorname{div}\left(\nabla_{\mathcal{F} c} s\right)=0
$$

where $\mathcal{F}^{c}$ is the complementary set of $\mathcal{F}$. As the gradient and divergence operators are local and linear, the formula above implies that whether a point needs to be included into $\mathcal{F}$ or not is something that can be evaluated locally at the neighbourhood of that point. In other words, the points that must be in $\mathcal{F}$ to get a perfect reconstruction or unpredictable points are those whose value cannot be predicted from their neighbours. Moreover, signal predictability is often considered a core concept for the analysis of complex systems $[1,2]$, by often considering measures of information growth such as e.g., Lyapunov exponents and Kolmogorov-Sinai entropy. We call the $\mathcal{F}_{u}$ set of unpredictable points the Unpredictable Points Manifold (UPM) of the signal, which is by definition the smallest set possible for having perfect eq. (13) reconstruction. Also, we are considering the hypothesis that $\mathcal{F}_{u}=\mathcal{F}_{\infty}$ for the signals under study, which is the basic conjecture giving rise to the 
framework of reconstructible systems [32]. Most practical experiences evidence that properly calculated MSCs are able to reconstruct the signals [37].

\section{Unpredictable point manifold (UPM) and its associated measures}

The concept of UPM-measure generalizes that of gradient measures introduced in the previous section. The first requirements for any singular positive UPM-measure $\mu$ are:

i) It is concerned with the local singularity structure of functions.

ii) It leads to a MSC as close to the UPM as possible.

In essence, we can think of a UPM-measure as a gradient measure that is sensitive to the local predictability degree, eq. (15). They represent the gradient form as finite differences in discrete signals and in a way that highlights unpredictability. Vector-valued wavelet projections are able to combine both features at the same time when carefully tuned.

Some standard singularity-analysis methods perform multiple wavelet projections of the measure to estimate the singularity exponents through log-log regressions on eq. (7).

However, projecting at several scales penalizes resolution - especially for the most singular structures [36] - and it increases computational complexity; that is why we concentrate on punctual estimates of the singularity exponents from the UPM measure $[23,36]$, namely:

$$
h(\mathbf{x})=\frac{\log \left(\mathcal{T}_{\Psi} \mu\left(\mathbf{x}, r_{0}\right) /\left\langle\mathcal{T}_{\Psi} \mu\left(\cdot, r_{0}\right)\right\rangle\right)}{\log r_{0}}+o\left(\frac{1}{\log r_{0}}\right)
$$

The denominator normalising the main logarithm $\left\langle\mathcal{T}_{\Psi} \mu\left(\cdot, r_{0}\right)\right\rangle$ is the expectation of the wavelet projection (in practice, its average over the whole signal or as wide as stationarity can be assumed) and lessens the relative amplitude of the fluctuations due to the $o\left(\frac{1}{\log r_{0}}\right)$ correction term. To effectively apply eq. (16) we require that $r_{0}$ is small enough so that the leading order dominates and we can neglect this correction term. The scale $r_{0}$ is thus the smallest accessible one, i.e., the pixel scale in practical terms. Without loss of generality, we conventionally assign a Lebesgue measure of 1 to the whole space domain, so for a $N_{1} \times N_{2} \times \cdots \times N_{d} d$-dimensional signal the value of $r_{0}$ is fixed to $r_{0}=\frac{1}{d \sqrt{N_{1} \cdots N_{d}}}$. This condition means that we require that the signal is large enough to make the first term in the right hand side of eq. (16) the dominant contribution to the singularity exponent. For practical purposes, any linear resolution $r_{0}$ finer than a hundredth is generally good enough.

\subsection{The Cross Fourier Transforms}

To maximise the attainable resolution of the singularity exponents function, we reduce the reconstruction formula, eq. (13, to the minimal meaningful neighbourhood of each point, i.e., its $2 d$ nearest neighbours in $2 d$ connexity neighbourhoods. These are expressed as the 4 neighbours at the ends of a cross for the case of $2 \mathrm{D}$ signals $(d=2)$ and that is why we talk about cross-gradient and gross-reconstruction operators.

In the general case and for any quantity $p(\mathbf{x})$, we represent the neighbourhood of a given point $\mathbf{x}_{0}$ by a $(2 d+1)$-component vector comprising this point and its nearest neighbours and following the indexing convention shown in Figure 1. The result is the vector $\left(p_{0}, \cdots, p_{2 d}\right)$.

The notion of local predictability directly extends to any dimension, independently of the number of neighbouring directions, with vectors of $(2 d+1)$ components linearly growing with the dimension $d$.

Let us remark the fact that the nearest connexity in such cross-like structuring remains inherent to our processing of the $\left(p_{0}, \cdots, p_{2 d}\right)$ vectors. We cannot just apply the standard harmonics of the Fourier Transform, i.e. $\left(e^{2 i k \pi /(2 d+1)}\right)_{k}$, as if they were standard linear vectors. Instead, since the centre of the cross and the position of all the other components are related to \pm 1 pixel displacements, the basic Nyquist frequency in each direction is $2 \pi / 3$. So that, the equivalent Fourier transform adapted to this cross formation has the following components:

$$
j=e^{2 \pi i / 3}=\cos (2 \pi / 3)+i \sin (2 \pi / 3)=-\frac{1}{2}+i \frac{\sqrt{3}}{2}, \bar{j}=j^{2}
$$

With them, we define the direct Cross Fourier Transform of any $(2 d+1)$-vector $\vec{p}=\left(p_{0}, \cdots, p_{2 d}\right)$ as the complex $(2 d+1)$-vector $\hat{\vec{p}}=\left(\hat{p}_{0}, \cdots, \hat{p}_{2 d}\right)$ obtained according to the following formula: 

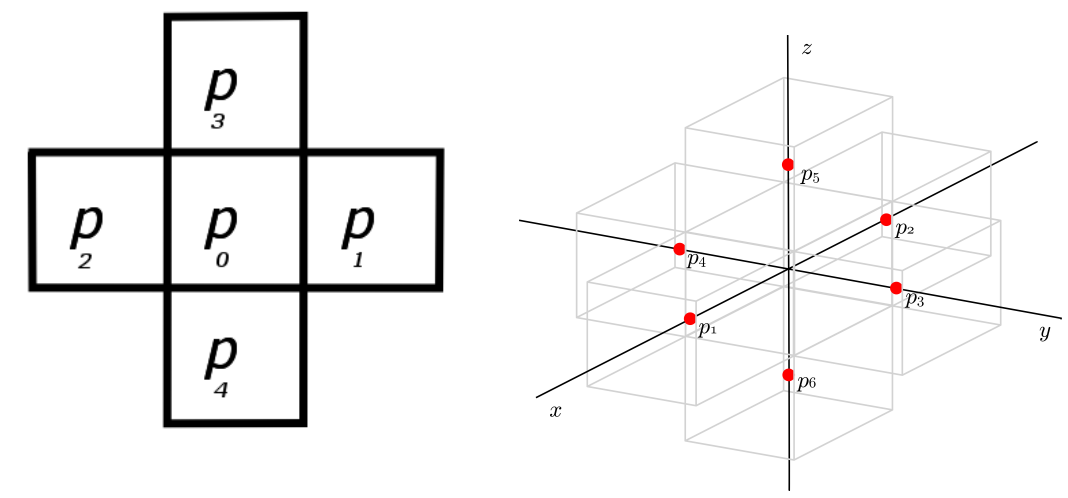

Figure 1. Schematic representation of the indexing of the points in the 2D cross (left) and the 3D case (right). The central point will be assigned the index 0 , the next point in the positive direction of the first coordinate axis will be indexed 1 , the opposite neighbour of it in the negative direction is indexed 2 , the next point in the second coordinate axis, positive direction is indexed as 3 , its opposite is indexed as 4 , and so on we proceed equivalently for any other coordinate axes.

$$
\hat{\vec{p}}=\mathbb{F} \vec{p}
$$

where $\mathbb{F}$ is the following $(2 d+1) \times(2 d+1)$ complex matrix:

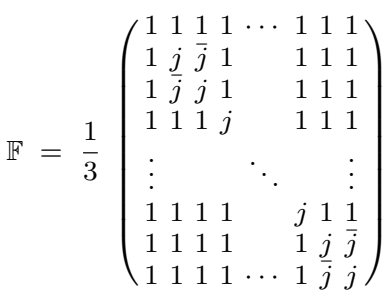

which represents the linear combination of the harmonics associated to the nearest cross-like displacements. The inverse of this matrix is given by:

$$
\mathbb{F}^{-1}=\left(\begin{array}{ccccccccc}
1-2 d & 1 & 1 & 1 & \cdots & 1 & 1 & 1 \\
1 & & j & j & 0 & & 0 & 0 & 0 \\
1 & & j & j & 0 & & 0 & 0 & 0 \\
1 & & 0 & 0 & \bar{j} & & 0 & 0 & 0 \\
\vdots & & & & \ddots & & & \vdots \\
1 & & 0 & 0 & 0 & & \bar{j} & 0 & 0 \\
1 & 0 & 0 & 0 & & 0 & \bar{j} & j \\
1 & & 0 & 0 & 0 & \cdots & 0 & j & j
\end{array}\right)
$$

We now introduce surrogates of the gradient and reconstruction operators restricted to the cross-like nearest neighbourhood. The goal is to use these to evaluate the local degree of predictability of the central point, and that is why they are based on the Cross Fourier Transform that we have just introudced.

The Cross Gradient Operator is the operator $\left(\partial_{k 1}, \cdots, \partial_{k d}\right)=\mathbb{F}^{-1} \cdot\left(\hat{\partial_{k 1}}, \cdots, \hat{\partial_{k d}}\right) \cdot \mathbb{F}$. When expressed in Fourier space, the operator is just a component-by-component multiplication of a given function by the $\hat{\partial_{k 1}} \cdots \hat{\hat{k}_{d}}$ factors, defined as:

$$
\hat{\partial_{k n}}=(0, \cdots, 0,0, \underbrace{i \sqrt{3},-i \sqrt{3}}_{2 n-1,2 n}, 0,0, \cdots, 0,0)
$$

where the index convention for the vectors runs from 0 to $2 d$ as before, and the index convention for the coordinate axes runs 1 to $d$ (as for $n$ ). 
The Cross Reconstruction Operator is one of the inverses of the Cross Gradient Operator (the gradient cancels any constant value so that the reconstruction is defined up to a constant). We canonically define it such that the resulting reconstructed vector is of zero mean along its $(2 d+1)$ components: $\sum_{i=0}^{2 d} s_{i}=0$. Therefore, the algorithm subtracts the mean of the vector as a first step (see the next subsection).

The Cross Reconstruction is the operator $R=\mathbb{F}^{-1} \cdot \hat{R} \cdot \mathbb{F}$ which in Fourier has $2 d$ functional components, namely $\hat{R}=\left(\hat{R}_{k 1}, \cdots, \hat{R}_{k d}\right)$. The reconstruction operator acts as the sum of all the products of each component by the corresponding components of the inputted gradient vector on which it operates:

$$
\hat{R}_{k n}=(0, \cdots, 0,0, \underbrace{-i / \sqrt{3}, i / \sqrt{3}}_{2 n-1,2 n}, 0,0, \cdots, 0,0)
$$

analogously to the gradient case. Also here their operation can be considered analogous to diagonal matrices acting on the $(2 d+1)$-dimensional $\hat{\vec{p}}$ vectors. The reconstructed vector is obtained as the sum of its component in each coordinate axis, from $k 1$ to $k d$.

The Cross Gradient and the Cross Reconstruction are the two basic algorithms for the design of the UPM-measures. Let us summarise again the algebraic structure we are considering: For every point in a $d$-dimensional, scalar, digital signal we have a

$(2 d+1)$-dimensional vector $\vec{p}$ made of the value at that point and its nearest neighbours. For every such vector we have a total of $d$ gradient components (which are cross-like structured $(2 d+1)$-vectors themselves), one for each dimension. For each one of these $d$ gradient components there is $(2 d+1)$-dimensional reconstruction operators and the sum of the resulting $d$ components results in a reconstructed $(2 d+1)$-vector. Cross-derivation followed by cross-reconstruction is equivalent to projection to a $(2 d+1)$-identity matrix (up to a normalisation constant in the 0-frequency in the cross-Fourier domain). The same procedure applies when the considered digital signal is vector-valued itself; then we just independently treat every component separate.

\subsection{A Local Correlation Singularity Measure}

One of the simplest and most generic ways of measuring the local unpredictability consists of just subtracting the signal value at a given point from the value inferred from its neighbour points. These values are previously detrended to cancel any global offset influence. The result then measures local correlation and so it is called the Local

Correlation Singularity Measure, which is calculated with the following algorithm: Goal: To evaluate $\mathcal{T}_{\Psi_{l c s m}} \mu\left(\mathbf{x}_{0}, r_{0}\right)$ at a given point $\mathbf{x}_{0}$ Algorithm:

(1) The nearest neighbourhood of $\mathbf{x}_{0}$ is expressed as a $(2 d+1)$-vector $\vec{s}=\left(s_{0}, \cdots, s_{2 d}\right)$ according to the cross scheme that we have presented in Figure 1.

(2) This vector is conveniently detrended: let $\bar{S}=\frac{1}{2 d-1} \sum_{i=0}^{2 d} s_{i}$, we define the detrended vector $\vec{p}=\left(p_{0}, \cdots, p_{2 d}\right)$ as:

$$
p_{0}=s_{0}+\bar{S} ; p_{i}=s_{i}-\bar{S}, i=1, \cdots, 2 d
$$

(3) We apply the Cross Gradient Operator to $\vec{p}$; the result is the gradient vectors $\vec{g}_{k n}$.

(4) The first component of these gradient vectors are kept for later use, $A_{n}=g_{k n, 0}$ (the zeroth/central component for the $k n$ axis direction), $n=1, \cdots, d$.

(5) We then set these components to zero, $g_{k 1,0}=\cdots=g_{k d, 0}=0$.

(6) We apply the Cross Reconstruction Operator to the resulting vectors $\vec{g}_{k n}$ and we sum them to obtain the reconstructed vector $\vec{r}$.

(7) We apply once more the Cross Gradient Operator on the reconstructed vector $\vec{r}$ to obtain $\vec{\rho}_{k n}$.

(8) We define the Local Correlation Singularity Measure as the modulus of the difference of the cross gradients at the centre of the cross, namely:

$$
\mathcal{T}_{\Psi_{l c s m}} \mu\left(\mathbf{x}_{0}, r_{0}\right)=\sqrt{\sum\left(A_{n}-\rho_{k n, 0}\right)^{2}}
$$

Which in fact is equivalent of keeping the modulus of a certain vector-valued wavelet projection. (9) Finally,we obtain the singularity exponent $h\left(\mathbf{x}_{0}\right)$ directly through eq. (16). 


\section{Most singular features in digital signals}

In the following, we show the application of the singularity analysis based on the Local Correlation Singularity Measure to empirical real-world signals of different dimensions.

We begin with a time series, i.e., a 1D case. It consists of the evolution of a stock market index: the Spanish IBEX-35 on a daily basis for 10 years. Singularity analysis has many applications in this case because the singularity exponents encode key aspects of the multiscale structure of the stock dynamics and identification of its MSC can be used not only in reconstruction but also in inference and stochastic forecast of future volatilities [24]. In Figure 2 we show the time series, its MSC and its reconstruction.
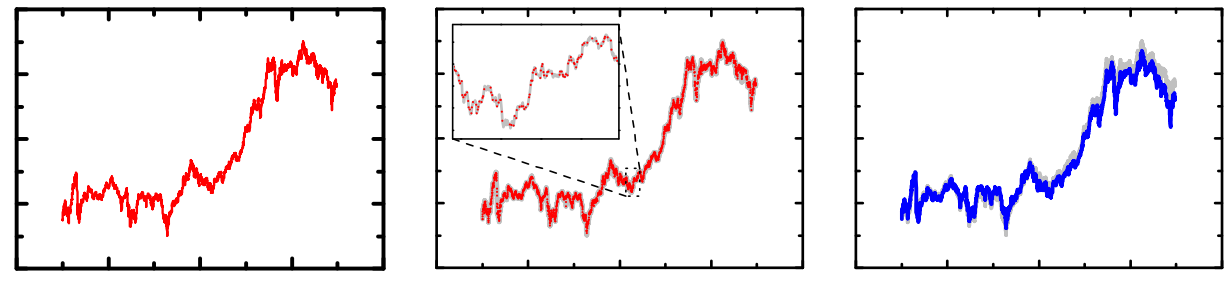

Figure 2. Left: Spanish blue chip index IBEX-35 opening daily values from 1990 to 2000. Centre: MSC points of this time series. When projected to the same discrete resolution of the original signal it covers somewhat less than $30 \%$ of the points. Right: Reconstructed time series from the gradient of the original signal on the MSC, using the reconstruction formula. The big part of the dynamics is recovered including most of its relevant aspects, resulting in a quality reconstruction of $27.9 \mathrm{~dB}$ as PSNR.

The Local Correlation Singularity Measure provides equally good results on 2D signals. We show examples of two different cases with different but related applications. The singularity exponents on natural images describe some kind of multiscale edges that provide a compact coding [32]. When applied on geophysical maps the objective consists in giving the descriptors of the turbulent dynamics as singularity exponents define eddies and filaments as well as they encode the interscale energy injection known as turbulent cascade process [37].

In Figure 3 we show an example of a natural scene and its reconstruction from its MSC. The singularity exponents are directly related to the local informativity degree and distribution of information in the image, as we can see when comparing them to the methodologically unrelated notion of multiscale entropy $[5,28]$ but with the advantage that they are sensibly finer and they are able to reach the resolution of 1 pixel, i.e., the same resolution of the original signal, Figure 4.

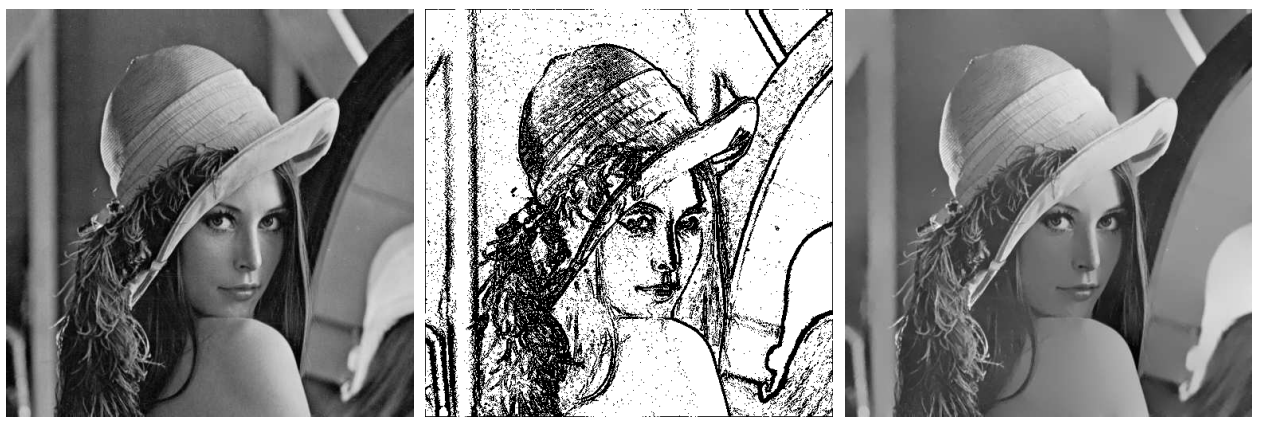

Figure 3. Left: Original Lena image. Centre: MSC, defined as $\{h<-0.5\}$; it comprises $30 \%$ of the points of the image. Right: Reconstruction. Though some of the finest details are missing due to the lack of capability of the method to capture every UPM point, the obtained reconstruction exhibits a fine quality of $24.5 \mathrm{~dB}$ PSNR.

In Figure 5 we show a case of a geophysical turbulence map: a Meteosat image in the infrared channel. Meteosat is a satellite specialised in the observation of clouds. Its infrared radiometry maps have two important advantages over visible: they are acquired 24 hours a day and they indicate the temperature of the clouds, thus permitting the identification of fronts and rain. Since clouds at a large scale are principally advected by the atmospheric flow, the structure of their global distribution describe the dynamics of atmospheric turbulence [4]. Our results in this case show that the MSC captures the most of the signal dynamics, as in the previous cases.

To illustrate a $3 \mathrm{D}$ case, we rather consider a $2 \mathrm{D}+1$ spatio-temporal signal, namely the time of evolution of 2D maps. Video constitutes a paradigm in that case and space-time correlations and transitions play a key role in the majority of their compression algorithms, but the spatial and temporal axis are not trivially comparable and isotropic methods need 

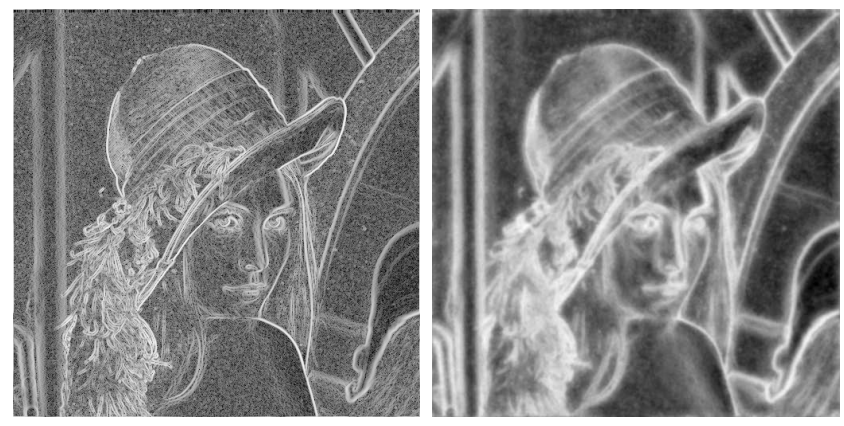

Figure 4. Left: Singularity exponents of the Lena image estimated using the Local Correlation Singularity Measure; they are represented using a inverse grayscale palette (the brightest the smaller, so more singular). Right: Multiscale entropy of the Lena image, in the same palette. All details of the detected features persist, confirming the correspondence between singularity exponents and degree of informativity. Resolution is nevertheless coarser for the entropy estimation.

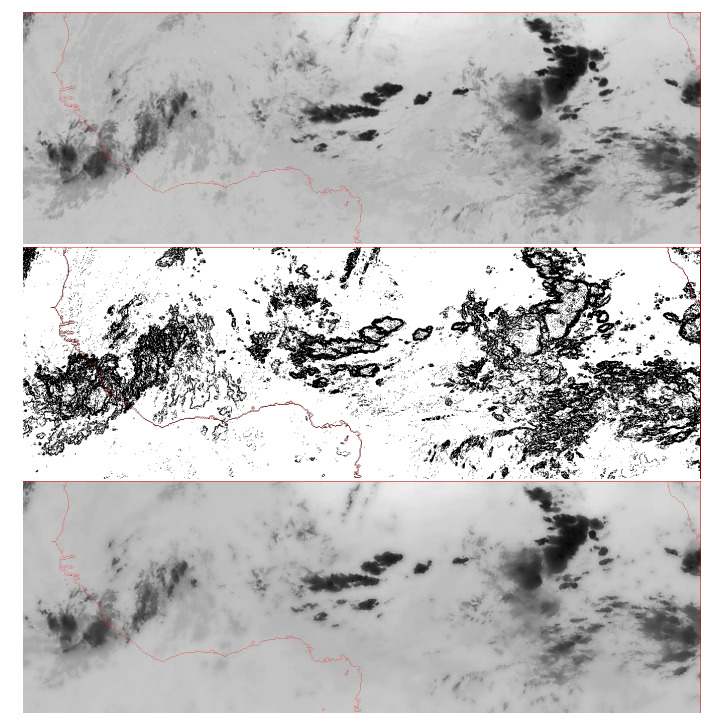

Figure 5. Top: Infrared radiance captured by one of the Meteosat satellites on 31st Jul 1998 on the area $20^{\circ} \mathrm{E}$ to $20^{\circ} \mathrm{W}$ and $0^{\circ}$ to $20^{\circ} \mathrm{N}$. Middle: MSC, defined as $\{h<-0.5\}$; it comprises $20 \%$ of the points of the image. Bottom: Reconstruction formula applied on the MSC points. Key aspects of atmospheric turbulence as the structure-rich subtropical winds are detected in the MSC and the reconstructed image reproduces them.

to be modified. Instead, we show a case of geophysical turbulence where typical velocities allow relating the spatial structure of turbulence to its temporal dynamics $[3,8,9]$. In Figure 6 we show several slices of a sequence showing the time evolution of global maps of air temperature at the atmospheric boundary layer. We also show the corresponding MSCs where singularity exponents have been evaluated on a space-time domain. This signal corresponds to the ERA-40 re-analysis product where global high resolution remote sensing variables are assimilated into an atmospheric level to estimate atmospheric circulation in a global and continuous way. We can see in the detail Figure 7 the comparison between the $2 \mathrm{D}$ singularity exponents, which take into account only the spatial structure, and the spatio-temporal singularity exponents, which appear more informative.

\section{Conclusions}

Having an accurate and robust estimation of the singularity exponents of a signal becomes essential in order to retrieve its key features and localise its information content, particularly in nonlinear and multiscale settings. Furthermore, the singularity degree as encoded by the local singularity exponent of a signal point is directly related to the exceptionality of that point in the signal or, in other words, how unpredictable it is or how difficult to reconstruct from the rest of the signal. In contrast, the regular, predictable points can be easily inferred from the singular ones, which means that the Most Singular Component (MSC) of the signal can be used for compact encoding, reconstruction or inference. 
10
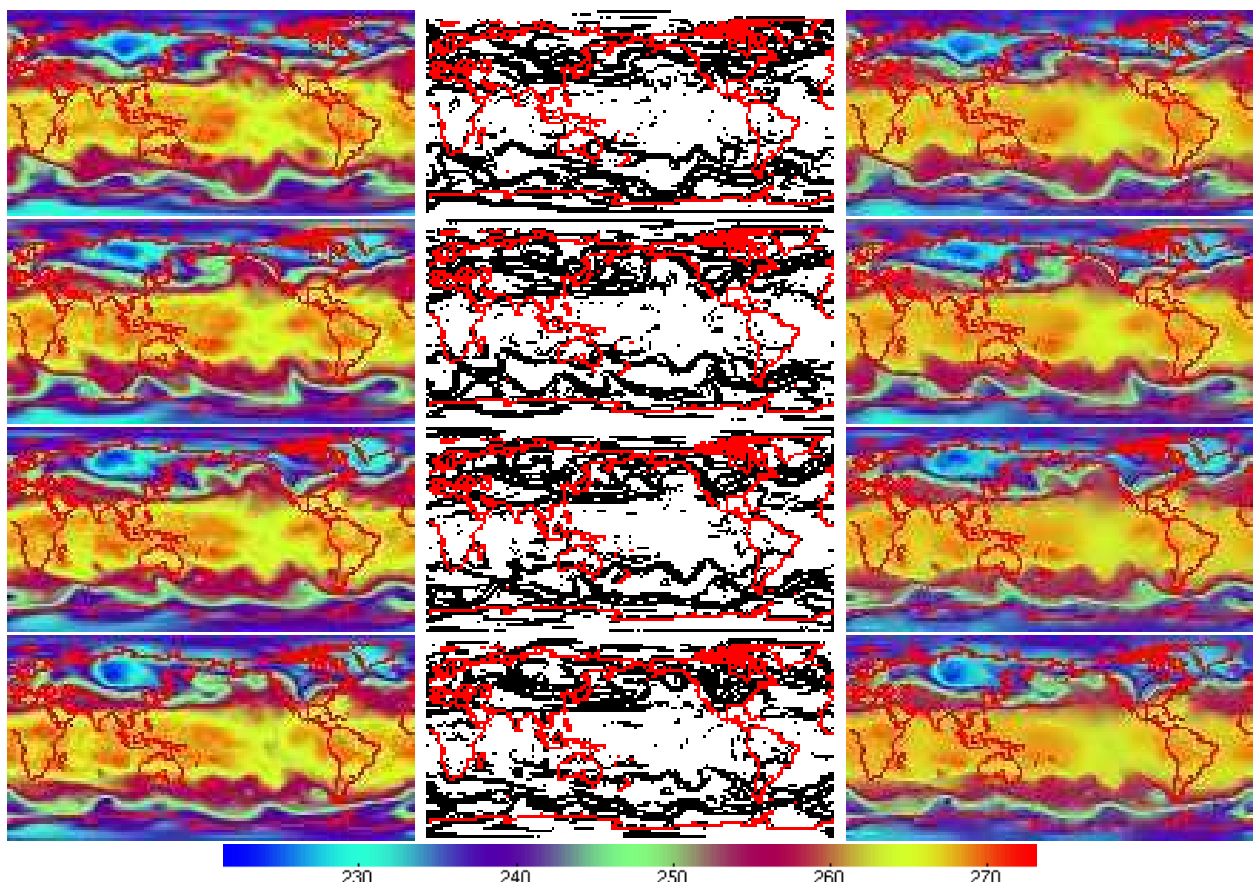

Figure 6. Left: ERA-40 re-analysis showing a sequence of global maps of the air temperature at the boundary layer of the atmosphere. Resolution is $2^{\circ} 5$ in space and $6 \mathrm{~h}$ in time. Temperature is expressed in Kelvins. Centre: MSC estimated from the singularity exponents in the spatio-temporal domain. Right: Reconstructions from the MSCs, capturing most of atmospheric circulation. Top to bottom: sequence slices at the 876576th, 876600th, 876624th and 876648th hour past midnight Jan 1st 1900.

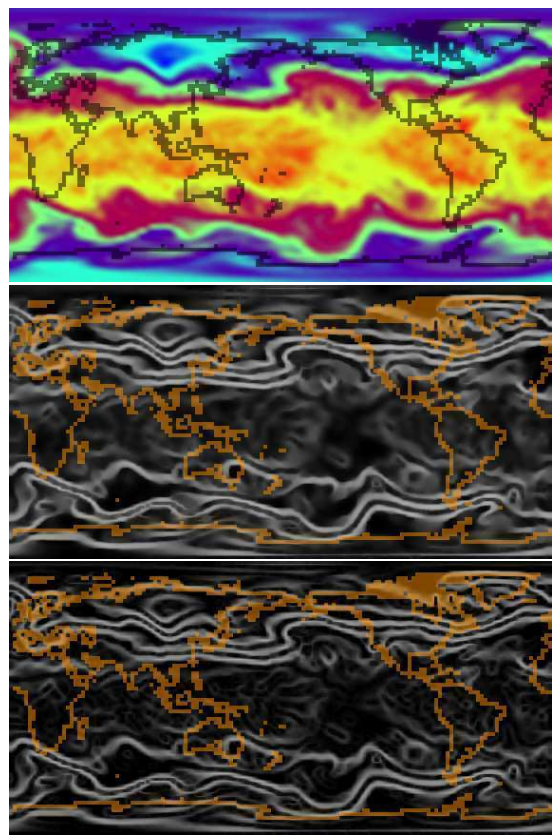

Figure 7. Top: 876576 th hour slice of ERA-40 artificially rescaled $4 \mathrm{x}$ with bicubic interpolation for the purpose of clarity of illustration. Middle: singularity exponents calculated only in the space. Some rescaling artefacts visibly appear but without significant disturbance of the fine structure details. Bottom: singularity exponents calculated in the space-time domain. Notice the increased degree of detail when the temporal information is taken into account. 
In this work, we have introduced an algorithm to accurately retrieve the singularity exponent at each point of a given signal. This algorithm calculates singularity exponents through a discrete mask, which is constructed as a measure of both the singularity degree and the local reconstructibility of that point. This mask actually represents a discretised numerical equivalent of a wavelet basis. With this basis, we introduce the notion of the Unpredictable Point Manifold (UPM) of a signal, which provides a rigorous definition for an intuitive concept: the degree of singularity of a given point in a signal is directly related to the degree of how exceptional is the value of the signal on that point compared to its direct neighbourhood.

Additionally, we have detailed a singularity-analysis algorithm based on the local correlations of the signal [31]. The algorithm introduces the so-called "Local Correlation Singularity Measure" as the basis to retrieve estimates of the singularity exponents with optimal accuracy and resolution. As a consequence, the signal can be reconstructed from its MSC points at high quality, which can be used in image compression [32], also in other related applications such as assessment of streamlines in turbulent flows [40,41], detection of meteorological convection [33] and identification of stock-market investment cycles [35].

We have shown the performance of the Local Correlation Singularity Measure algorithm on empirical signals of different types. We have illustrated a 1D case with stock-market series, where estimation of the UPM allows inference and forecasting methods [24], the 2D cases of natural scenes and the atmospheric dynamics as seen by Meteosat IR maps, and a $3 \mathrm{D}$ case of the type $2 \mathrm{D}$-space+1D-time, being careful with the space-time correspondence, illustrated with a sequence of global air temperature maps from the ERA-40 global climate re-analysis, where we have observed how the singularity exponents taken in the spatio-temporal domain give more details in that case than the $2 \mathrm{D}$ singularity exponents evaluated independently.

\section{Acknowledgements}

The authors acknowledge financial support from Fonds LEDUCQ, the ARC FIBAUR project and project MIDAS-6 AYA2010-22062-C05.

\section{References}

[1] E. Aurell, G. Boffetta, A. Crisanti, G. Palading, and A. Vulpiani, Predictability in the large: an extension of the concept of lyapunov exponent, Journal of Physics A 30 (1997), pp. 1-26.

[2] G. Boffetta, M. Cencini, M. Falcioni, and A. Vulpiani, Predictability: a way to characterize complexity, Physics reports 356 (2001), pp. 367-474.

[3] H. Chaté and P. Manneville, Transition to turbulence via spatio-temporal intermittency, Phys. Rev. Lett. 58 (1987), pp. 112-115.

[4] Y. Chigirinskaya, D. Schertzer, S. Lovejoy, A. Lazarev, and A. Ordanovich, Unified multifractal atmospheric dynamics tested in the tropics part 1: horizontal scaling and self organized criticality, Nonlinear Processes in Geophysics 1 (1994), pp. 105-114

[5] M. Costa, A.L. Goldberger, and C.K. Peng, Multiscale entropy analysis of complex physiologic time series, Phys. Rev. Lett. 89 (2002), p. 068102.

[6] I. Daubechies, Ten Lectures on Wavelets, Cbms-Nsf Regional Conference Series in Applied Mathematics, SIAM: Society for Industrial and Applied Mathematics, Montpelier, Vermont (1992).

[7] K. Falconer, Fractal Geometry: Mathematical Foundations andApplications, John Wiley and sons, Chichester (1990).

[8] U. Frisch, Turbulence: The legacy of A.N. Kolmogorov, Cambridge Univ. Press, Cambridge MA (1995).

[9] T. Houra and Y. Nagano, Spatio-temporal turbulent structures of thermal boundary layer subjected to non-equilibrium adverse pressure gradient, International Journal of Heat and Fluid Flow 29 (2008), pp. 591 - 601, the Fifth International Symposium on Turbulence and Shear Flow Phenomena (TSFP5).

[10] J. Isern-Fontanet, A. Turiel, E. Garcia-Ladona, and J. Font, Microcanonical multifractal formalism: application to the estimation of ocean surface velocities, Journal of Geophysical Research 112 (2007), p. C05024.

[11] S. Jaffard, Multifractal formalism for functions. I. Results valid for all functions, SIAM Journal of Mathematical Analysis 28 (1997), pp. 944-970.

[12] C.L. Jones, G.T. Lonergan, and D.E. Mainwaring, Wavelet packet computation of the hurst exponent, J. Phys. A: Math. Gen. 29 (1996), p. 2509.

[13] M.S. Lee, Signal smoothness estimation in hölder spaces, International Journal of Computer Mathematics 73 (2000), pp. 321-332.

[14] M.S. Lee, Noisy image enhancement on smooth function spaces, International Journal of Computer Mathematics 85 (2008), pp. 1463-1472.

[15] S. Mallat, A Wavelet Tour of Signal Processing, Academic Press, 2nd Edition (1999).

[16] S. Mallat and W.L. Huang, Singularity detection and processing with wavelets, IEEE Trans. in Inf. Th. 38 (1992), pp. 617-643.

[17] S. Mallat and S. Zhong, Wavelet transform maxima and multiscale edges, in Wavelets and their applications, M.B.R. et al, ed., Jones and Bartlett, Boston, 1991. 
[18] S. Mallat and S. Zhong, Characterization of signals from multiscale edges, IEEE Trans. on Pattern Analysis and Machine Intelligence 14 (1992), pp. 710-732.

[19] J.F. Muzy, E. Bacry, and A. Arneodo, Wavelets and multifractal formalism for singular signals: Application to turbulence data, Physical Review Letters 67 (1991), pp. 3515-3518.

[20] G. Parisi and U. Frisch, On the singularity structure of fully developedturbulence, in Turbulence and Predictability in Geophysical Fluid Dynamics. Proc. Intl. School of Physics E. Fermi, North Holland, Amsterdam, 1985, pp. 84-87.

[21] O. Pont, A. Turiel, and C.J. Pérez-Vicente, Empirical evidences of a common multifractal signature in economic, biological and physical systems, Physica A 388 (2009), pp. 2025-2035.

[22] O. Pont, A. Turiel, and C.J. Pérez-Vicente, On optimal wavelet bases for the realization of microcanonical cascade processes, Int. J. Wavelets Multi., IJWMIP 9 (2011), pp. 35-61.

[23] O. Pont, A. Turiel, and C.J. Pérez-Vicente, Application of the microcanonical multifractal formalism to monofractal systems, Physical Review E 74 (2006), pp. 061110-061123.

[24] O. Pont, A. Turiel, and C.J. Pérez-Vicente, Description, modeling and forecasting of data with optimal wavelets, Journal of Economic Interaction and Coordination 4 (2009), pp. 39-54.

[25] O. Pont, A. Turiel, and H. Yahia, An optimized algorithm for the evaluation of local singularity exponents in digital signals, in 14th International Workshop, IWCIA 2011, Lecture Notes in Computer Science (LNCS), vol. 6636, Springer, Madrid, Spain, 2011, pp. 346-357.

[26] Z.S. She and E. Leveque, Universal scaling laws in fully developed turbulence, Physical Review Letters 72 (1994), pp. 336-339.

[27] I. Simonsen, A. Hansen, and O. Magnar, Determination of the hurst exponent by use of wavelet transforms, Phys. Rev. E 58 (1998), pp. 2779-2787.

[28] J.L. Starck and F. Murtagh, Multiscale entropy filtering, Signal Processing 76 (1999), pp. 147 - 165.

[29] Z.R. Struzik, Determining local singularity strengths and their spectra with the wavelet transform, Fractals 8 (2000), pp. 163-179.

[30] A. Turiel, Relevance of multifractal textures in static images, Electronic Letters on Computer Vision and Image Analysis 1 (2003), pp. 35-49.

[31] A. Turiel, Method and system for the singularity analysis of digital signals (2008), patent registered under number PCT/ES2008/070195.

[32] A. Turiel and A. del Pozo, Reconstructing images from their most singular fractal manifold, IEEE Trans. on Im. Proc. 11 (2002), pp. 345-350.

[33] A. Turiel, J. Grazzini, and H. Yahia, Multiscale techniques for the detection of precipitation using thermal IR satellite images, IEEE Geoscience and Remote Sensing Letters 2 (2005), pp. 447-450, doi:10.1109/LGRS.2005.852712.

[34] A. Turiel and N. Parga, The multi-fractal structure of contrast changes innatural images: from sharp edges to textures, Neural Computation 12 (2000), pp. 763-793.

[35] A. Turiel and C. Pérez-Vicente, Role of multifractal sources in the analysis of stock market time series, Physica A 355 (2005), pp. 475-496.

[36] A. Turiel, C. Pérez-Vicente, and J. Grazzini, Numerical methods for the estimation of multifractal singularity spectra on sampled data: a comparative study, Journal of Computational Physics 216 (2006), pp. 362-390.

[37] A. Turiel, H. Yahia, and C. Pérez-Vicente, Microcanonical multifractal formalism: a geometrical approach to multifractal systems. Part I: Singularity analysis, Journal of Physics A 41 (2008), p. 015501.

[38] A. Turiel, G. Mato, N. Parga, and J.P. Nadal, The self-similarity properties of natural images resemble those of turbulent flows, Physical Review Letters 80 (1998), pp. 1098-1101.

[39] A. Turiel, J. Isern-Fontanet, E. García-Ladona, and J. Font, Multifractal method for the instantaneous evaluation of the stream function in geophysical flows, Physical Review Letters 95 (2005), p. 104502, doi:10.1103/PhysRevLett.95.104502.

[40] A. Turiel, J. Solé, V. Nieves, J. Ballabrera-Poy, and E. García-Ladona, Tracking oceanic currents by singularity analysis of micro-wave sea surface temperature images, Remote Sensing of Environment 112 (2008), pp. 2246-2260.

[41] A. Turiel, V. Nieves, E. García-Ladona, J. Font, M.H. Rio, and G. Larnicol, The multifractal structure of satellite temperature images can be used to obtain global maps of ocean currents, Ocean Science 5 (2009), pp. 447-460. 